Article

\title{
Isolation and Analysis of Anthocyanin Pathway Genes from Ribes Genus Reveals MYB Gene with Potent Anthocyanin-Inducing Capabilities
}

\author{
Pavel Starkevič ${ }^{1,2}$, Aušra Ražanskienè ${ }^{1}$, Urtė Starkevič ${ }^{1}$, Vaiva Kazanavičiūtè ${ }^{1}$, \\ Erna Denkovskienè ${ }^{1}$, Vidmantas Bendokas ${ }^{3}$, Tadeušas Šikšnianas ${ }^{3}$, Rytis Rugienius ${ }^{3}{ }^{\circledR}$, \\ Vidmantas Stanys ${ }^{3}$ and Raimundas Ražanskas ${ }^{1, *(\mathbb{D})}$ \\ 1 Department of Eukaryotic Gene Engineering, Institute of Biotechnology, Vilnius University, \\ 10257 Vilnius, Lithuania; pavel.visarcuk@gmail.com (P.S.); ausra.razanskiene@bti.vu.lt (A.R.); \\ star.urte@gmail.com (U.S.); kaaiva@yahoo.de (V.K.); erna@nomadsbio.lt (E.D.) \\ 2 Nature Research Centre, Akademijos str. 2, 08412 Vilnius, Lithuania \\ 3 Department of Orchard Plant Genetics and Biotechnology, Institute of Horticulture, Lithuanian Research \\ Centre for Agriculture and Forestry, 54333 Babtai, Lithuania; v.bendokas@1sdi.lt (V.B.); \\ t.siksnianas@lsdi.lt (T.Š.); r.rugienius@lsdi.lt (R.R.); v.stanys@1sdi.lt (V.S.) \\ * Correspondence: raimundas.razanskas@bti.vu.lt
}

Received: 24 July 2020; Accepted: 19 August 2020; Published: 22 August 2020

\begin{abstract}
Horticultural crops of the Ribes genus are valued for their anthocyanin-rich fruits, but until now, there were no data about the genes and regulation of their flavonoid pathway. In this study, the coding sequences of flavonoid pathway enzymes and their putative regulators MYB10, bHLH3 and WD40 were isolated, and their expression analyzed in fruits with varying anthocyanin levels from different cultivars of four species belonging to the Ribes genus. Transcription levels of anthocyanin synthesis enzymes and the regulatory gene RrMYB10 correlated with fruit coloration and anthocyanin quantities of different Ribes cultivars. Regulatory genes were tested for the ability to modulate anthocyanin biosynthesis during transient expression in the leaves of two Nicotiana species and to activate Prunus avium promoters of late anthocyanin biosynthesis genes in N. tabacum. Functional tests showed a strong capability of RrMyb10 to induce anthocyanin synthesis in a heterologous system, even without the concurrent expression of any heterologous bHLH, whereas RrbHLH3 enhanced MYB-induced anthocyanin synthesis. Data obtained in this work facilitate further analysis of the anthocyanin synthesis pathway in key Ribes species, and potent anthocyanin inducer RrMyb10 can be used to manipulate anthocyanin expression in heterologous systems.
\end{abstract}

Keywords: anthocyanins; Ribes nigrum; Ribes uva-crispa; Ribes rubrum; MYB10; bHLH

\section{Introduction}

Ribes, the only genus of the Grossulariceae family, contains woody perennial plants native throughout temperate regions of the Northern Hemisphere. The first members of the genus were domesticated 400-500 years ago, and today descendants of at least eighteen species are widely cultivated in Europe and, to a smaller degree, in China, New Zealand and North and South America [1]. The most commercially important species are blackcurrant (Ribes nigrum L.) and redcurrant (Ribes rubrum L.). Other domesticated currants and gooseberries are commonly cultivated in home gardens, but also have some commercial value. According to the Food and Agriculture Organization of the United Nations (http://www.fao.org/faostat/en/\#data/QC), the annual worldwide production of currants in 2018 was about 660,000 tons (almost all in Europe, nearly 90\% in Eastern Europe), and the production of gooseberries surpassed 170,000 tons (almost all in Europe). Small fleshy fruits produced by 
numerous cultivars of the genus, especially blackcurrants, are considered an important source of health-promoting phytochemicals, possessing immunomodulatory, anti-inflammatory, antimicrobial and cancer prevention potential [2,3]. These valuable properties of blackcurrants are attributed mainly to high quantities of vitamin C (reaching over $350 \mathrm{mg} / 100 \mathrm{~g}$ fresh weight) and phenolic compounds, mainly anthocyanins (reaching over $500 \mathrm{mg} / 100 \mathrm{~g}$ fresh weight) $[2,4,5]$. Besides possessing strong antioxidant and other health-promoting qualities, anthocyanins are also the main components determining the color of berries, an important commercial feature $[3,6]$.

More than 600 different anthocyanin structures are known, and at least fifteen of them have been found in blackcurrant [7]. Several studies aimed to determine the composition and quantities of anthocyanins in different cultivars of currants and gooseberries and revealed the predominance of cyanidin-3-O-rutinoside, delphinidin-3-O-rutinoside, cyanidin-3-O-glucoside and delphinidin-3-O-glucoside $[4,8,9]$. The key intermediates and enzymes leading to the production of different anthocyanin classes are already well investigated in a model plant, Arabidopsis thaliana, and many crop species [10-12]. The spectrum of anthocyanin molecules synthesized in Ribes fruits suggests a scheme of an anthocyanin synthesis pathway with an inactive pelargonidin branch (Figure 1), which is a subset of the general flavonoid pathway [13].

The main regulators of anthocyanin synthesis in different plant tissues are MYB R2R3 class transcription factors, which act in concert with bHLH and WD40 proteins by forming the so-called MBW complex [14]. Based on gene expression studies of Arabidopsis and some other plants, the flavonoid biosynthetic pathway is subdivided into "early" (chalcone synthase (CHS), chalcone isomerase (CHI), flavanone 3-hydroxylase (F3H)) and "late" (dihydroflavonol 4-reductase (DFR), anthocyanin synthase (ANS), UDP-glucose:flavonoid glucosyltransferase (UFGT)) genes. It was established that the MBW complex regulates the expression of late genes, but the presence of this complex is not required for the expression of early genes. However, the exact late and early sets differ between species and even tissues, and they are determined by interactions between different MYB and bHLH proteins, while plentiful MYB variants are responsible for the fine-tuning of pigment production [15]. Recently, the expressions of the anthocyanin biosynthetic pathway and related genes have been studied through global transcriptome methods, uncovering a wealth of information in important fruit crops, such as sweet cherry, bayberry and black raspberry [16-18]. These studies confirmed that anthocyanin production correlates with the expression levels of the main structural genes and some MYB proteins, although the presence of early and late gene sets was not always discernible.

Despite the importance of anthocyanins and intensive studies of their composition and properties, until now, there were no data about flavonoid pathway genes and their regulation in berries of the Ribes genus. The whole Saxifragales clade is not studied at the molecular level as extensively as, for example, the asterid and rosid clades, no genomes are sequenced and not much overall sequence information is available. The only exception now is the genus Paeonia, with the expression of anthocyanin pathway genes analyzed in P. lactiflora and P. suffruticosa flowers [19]. However, anthocyanin content in Paeonia flowers differs significantly from that of Ribes fruits, with peonidin, pelargonidin and cyanidin 3- and 3,5-glucosides being the main species in Paeonia [20]. Although anthocyanin-regulating MYBs and, to a lesser degree, the other regulators have been analyzed in many plants, such genes in Saxifragales were only recently identified in P. suffruticosa [21-23].

In the present study, our aim was to isolate anthocyanin pathway genes and their regulators, to analyze their expression in fruits of different Ribes species and their cultivars to identify ones associated with anthocyanin levels and to test the anthocyanin-inducing capabilities of isolated transcription factors in a heterologous expression system. 

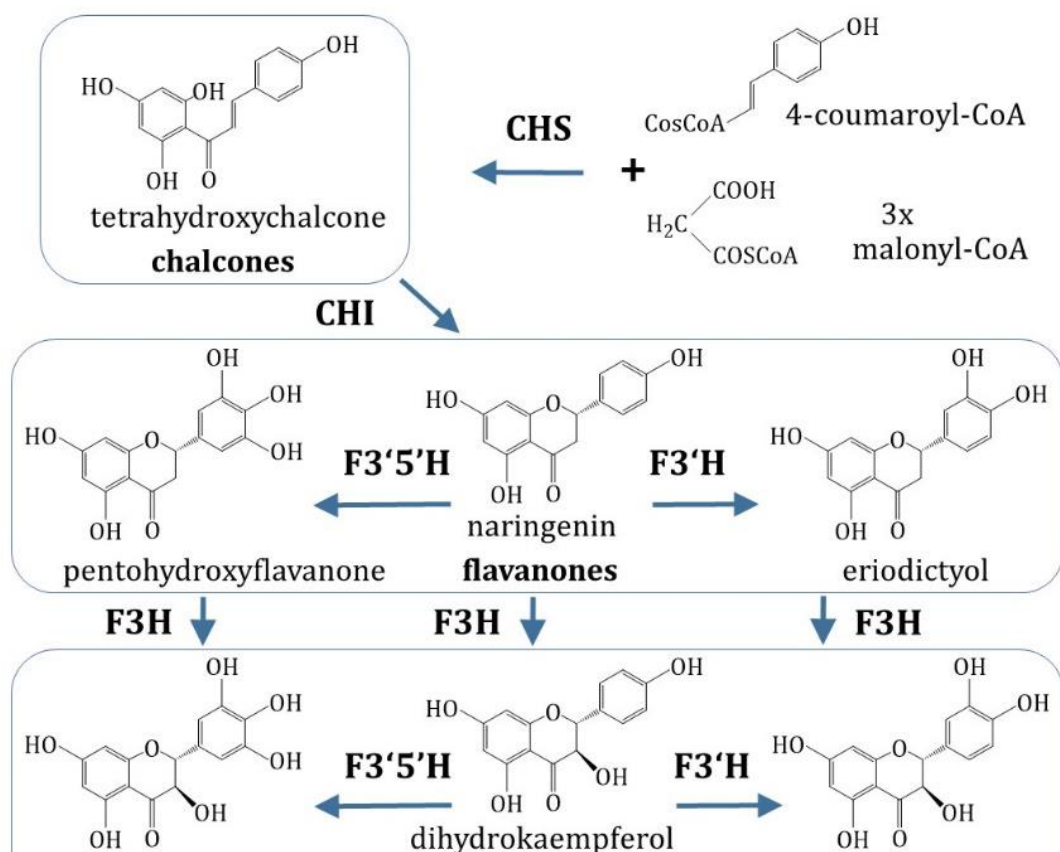
dihydromyricetin dihydroflavonols dihydroquercetin

leucodelphinidin $\quad$ leucopelargonidin

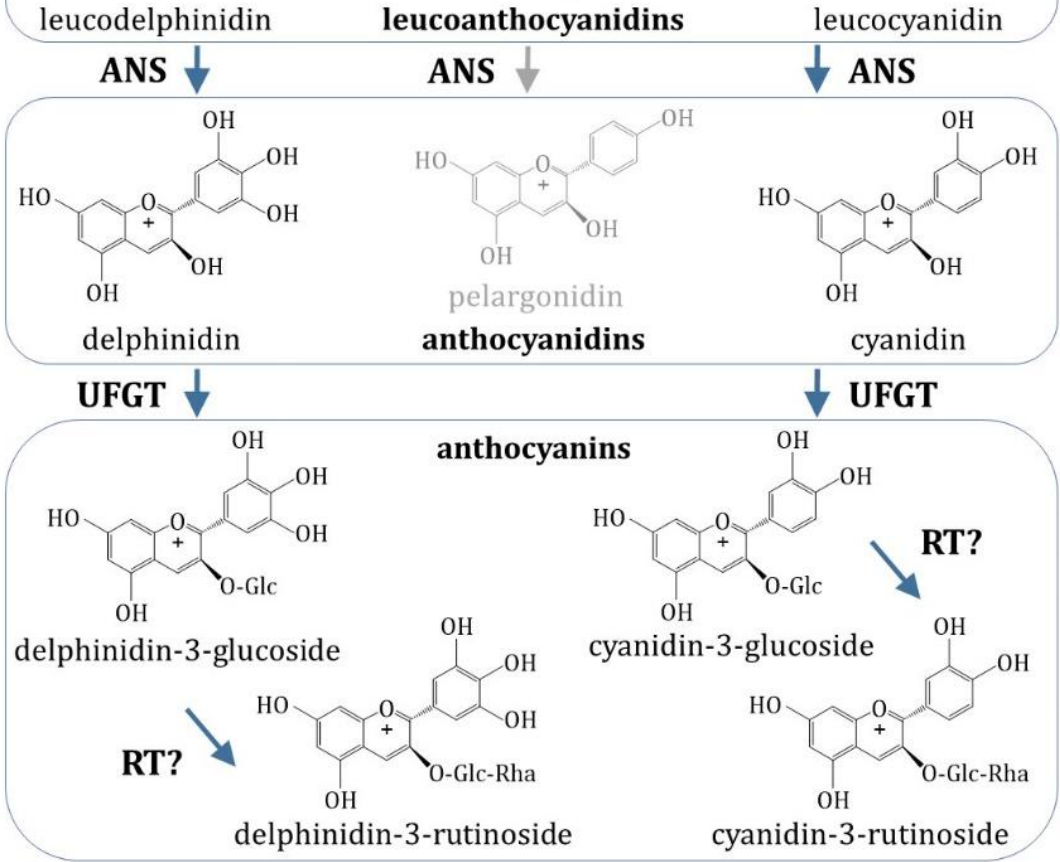

Figure 1. Putative anthocyanin pathway in fruits of the Ribes genus. Only the branches leading to the most prevalent Ribes anthocyanins, delphinidin-3-O-rutinoside and cyanidin-3-O-rutinoside, are completely shown. Abbreviations of enzymes: CHS, chalcone synthase; $\mathrm{CHI}$, chalcone isomerase; $\mathrm{F} 3 \mathrm{H}$, flavonoid 3-hydroxylase; F3' $\mathrm{H}$, flavonoid 3'-hydroxylase; F3'5' $\mathrm{H}$, flavonoid 3', 5'-hydroxylase; DFR, dihydroflavonol reductase; ANS, anthocyanidin synthase; UFGT, UDP-glucose:flavonoid 3-O-glucosyl transferase; RT, putative rhamnosyltransferase. 


\section{Results}

\subsection{Isolation of Anthocyanin Pathway Genes from Four Ribes Species}

A PCR with a degenerated primers approach was used to clone genomic and cDNA fragments from different Ribes species. Since the genus Ribes belongs to the Saxifragales taxon, for which no sequence information on flavonoid pathway genes was available at the start of this work, the primers were designed to target most conservative regions in all known relevant gene sequences, mainly from the rosid and asterid taxa. The sequences were cloned from mature fruits of cultivars with the highest fruit anthocyanin content: R. nigrum "Ben Tirran", R. uva-crispa "Chorny negus", R. rubrum "Jonkheer van Tets" and R. aureum "Cordona'". After sequence information for the Ribes gene fragment was obtained, $3^{\prime}$ - and $5^{\prime}$-RACE (rapid amplification of cDNA ends) was applied to clone full or partial sequences of the main anthocyanin pathway enzymes UFGT, ANS, DFR, F3H, F3' H, F3' $5^{\prime} \mathrm{H}, \mathrm{CHI}$ and $\mathrm{CHS}$, as well as transcriptional regulators MYB10, bHLH3 and WD40. To gain the sequence information needed to design Ribes cross-species qPCR primers, 200-300 bp fragments were also sequenced from other analyzed cultivars.

Sequence comparisons revealed that enzymes of different Ribes species share close homology (>90\% of identical residues) at both the amino acid and nucleotide level. Noncoding flanking sequences differ much more and have insertions-deletions. The similarity between Ribes and Paeonia for some enzymes (ANS, F3H) is $83-88 \%$ of identical residues at the amino acid level and $77-81 \%$ at the nucleotide level, whereas the similarity of Ribes sequences to enzymes of species belonging to the rosid, asterid and caryophyllales clades is generally lower. Meanwhile, the similarity between other Ribes and Paeonia enzymes is not as high, probably because different variants of the same enzymes are expressed in Ribes fruits and Paeonia petals, the sources of the analyzed sequences (Figures S1-S9).

The similarity of Ribes flavonoid pathway enzymes to the counterparts from other species can be best exemplified by comparing ANS sequences (Figure 2). Sequence information for ANS is available from many species and, based on the available sequence data, it can be hypothesized that many plant species possess only one functional ANS gene per haploid genome [16-18]. This hypothesis is indirectly supported by the fact that ANS mutants, such as white pomegranate and yellow raspberry cultivars, as well as an A. thaliana mutant, do not synthesize anthocyanins in any tissues, in any stage of development or in response to any environmental factors [24-26]. As can be seen in the phylogenetic timetree (Figure 2A), Ribes ANS protein sequences are more homologous to the sequences of rosids than to the asterid clade, and sequences inside the asterid branch are more divergent, although that is not true for sequences of all anthocyanin pathway enzymes. More sequence comparisons with percentages of identical amino acids and nucleotides to R. nigrum ANS are depicted by boxplots (Figure 2B) and the phylogenetic trees of main pathway enzymes are presented in supplementary figures.

Anthocyanin pathway genes are regulated by a ternary complex MBW, which includes WD40 and proteins from the bHLH and MYB families. In this work, we tried to clone all three members of this regulatory complex. The sequence of the only constant member of this complex, WD40, is highly conservative, and this was also confirmed with Ribes. The R. nigrum WD40 protein shares at least 75\% identical residues with any known counterpart from the asterid and rosid clades (Figure S7). 

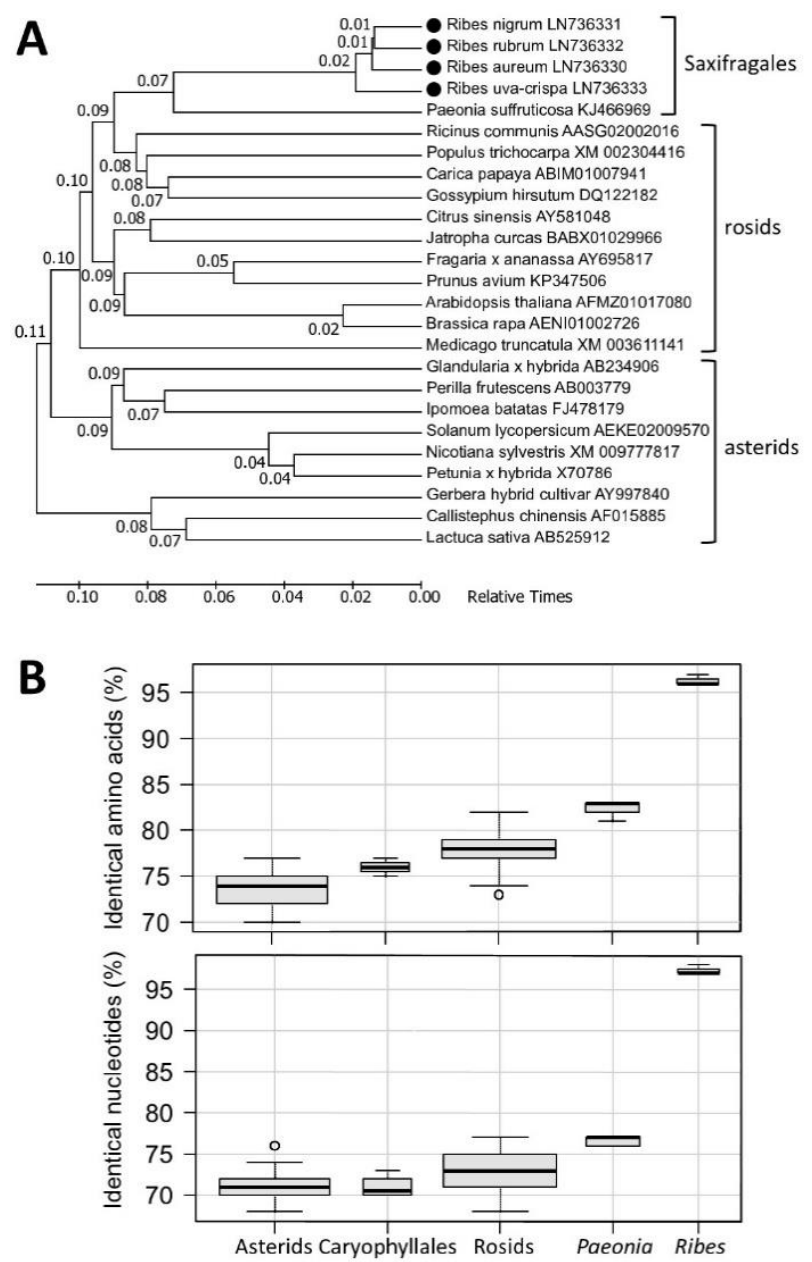

Figure 2. Similarity between ANS sequences from Ribes genus and other taxa. (A) A phylogenetic timetree of selected ANS protein sequences. Divergence times were calculated with the RelTime method using MEGA7. All proteins are named with the species name and accession number. (B) Boxplots showing the similarity between ANS sequences. Each boxplot shows the spread of percentages of identical residues in pairwise alignments between $R$. nigrum ANS and other ANS sequences. In each group (except Ribes and Paeonia) each sequence aligned to $R$. nigrum ANS represents a different genus. The number of pairwise alignments in each group: asterids, 21; caryophyllales, 4; rosids, 22; Paeonia, 5; Ribes, 4 .

\subsection{Anthocyanin-Regulating Genes of Ribes Genus}

Since the bHLH3 protein is the most obvious activating bHLH member of the MBW complex, it was cloned from the Ribes genus in this work. As can be seen in the phylogenetic timetree (Figure 3A), the cloned sequence from $R$. rubrum clearly belongs to the bHLH3/TT8 branch of bHLH group IIIf proteins. Proteins belonging to different Saxifragales genera are positioned inside the rosid clade according to the created phylogenetic tree. The sequence logo with marked structural elements and known important amino acids (Figure 3B) shows that the cloned cDNA encodes all features needed to make functionally active bHLH3 protein. 
A

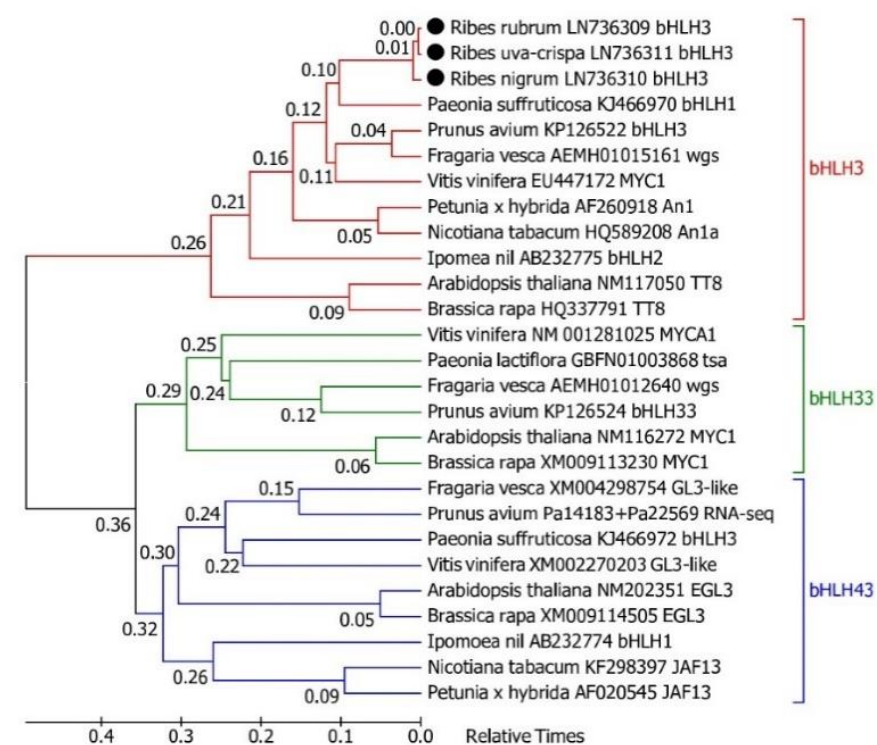

B

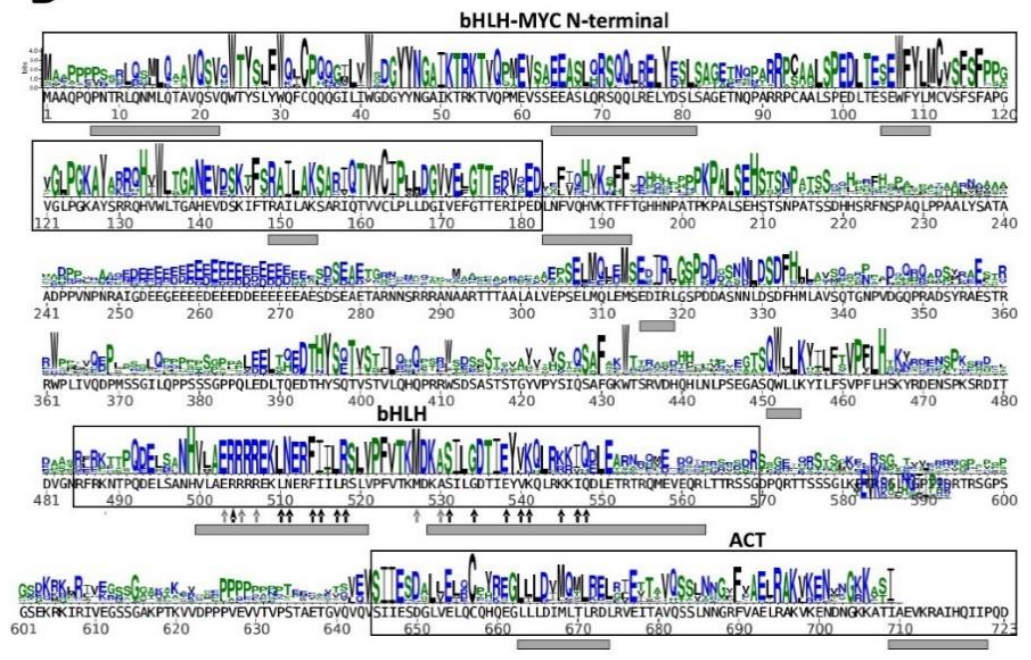

Figure 3. Ribes rubrum bHLH3 is homologous to other bHLH3-TT8 proteins. (A) A phylogenetic timetree of selected bHLH sub-group IIIf protein sequences. All proteins named with species name, accession number and original protein name. Sources of unnamed sequences: wgs, genes identified from NCBI Whole Genome Sequencing database; tsa, sequences identified from NCBI Transcriptome Shotgun Assembly sequence database; RNA-seq, cDNA from whole transcriptome study. (B) Sequence logo representing an alignment of $80 \mathrm{bHLH3}$-like proteins from different species of the Pentapetalae taxonomic branch. Empty boxes denote protein domains as defined in NCBI's Conserved Domain Database. Gray boxes beneath the sequence specify helixes as predicted by PSIPRED. Gray arrows point to amino acids involved in DNA binding; black arrows point to amino acids involved in the dimerization interface; black arrow with long head points to amino acid essential to E-box specificity.

The last member of the MBW complex is a protein from the ubiquitous MYB family. In this work, we aimed to clone the gene encoding R2R3 class subgroup 6 protein, homologous to the group named MYB10 in Rosaceae and some other species. A phylogenetic tree composed of arbitrary selected subgroup 6 MYB proteins (Figure 4A) places Ribes sequences together with other anthocyanin-regulating proteins, but not with MYB proteins regulating other branches of the flavonoid pathway. The sequence logo (Figure 4B) shows that all important functional and structural elements of anthocyanin-regulating MYB proteins are present in the Ribes MYB10 sequences, and the presence of motif 6 places them in subgroup 6 of the MYB superfamily. 
A

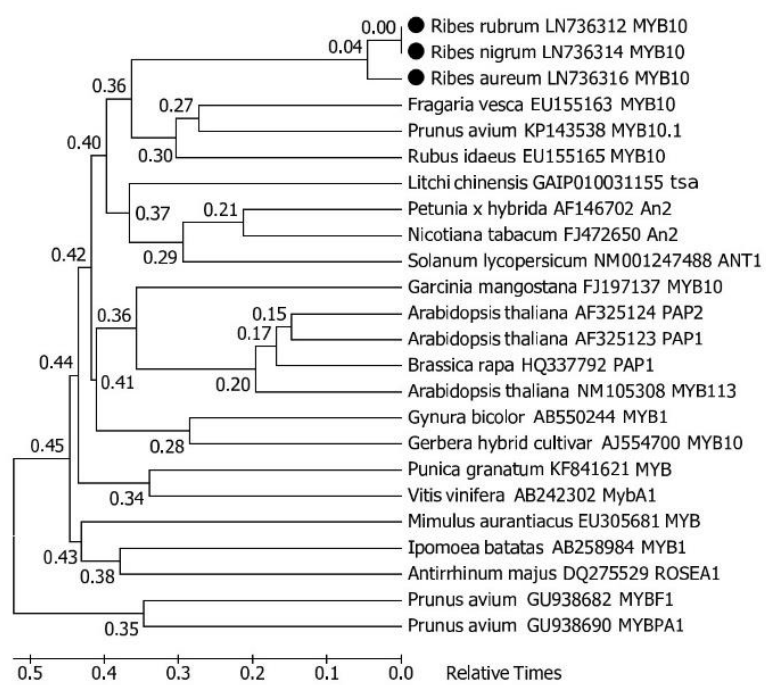

B
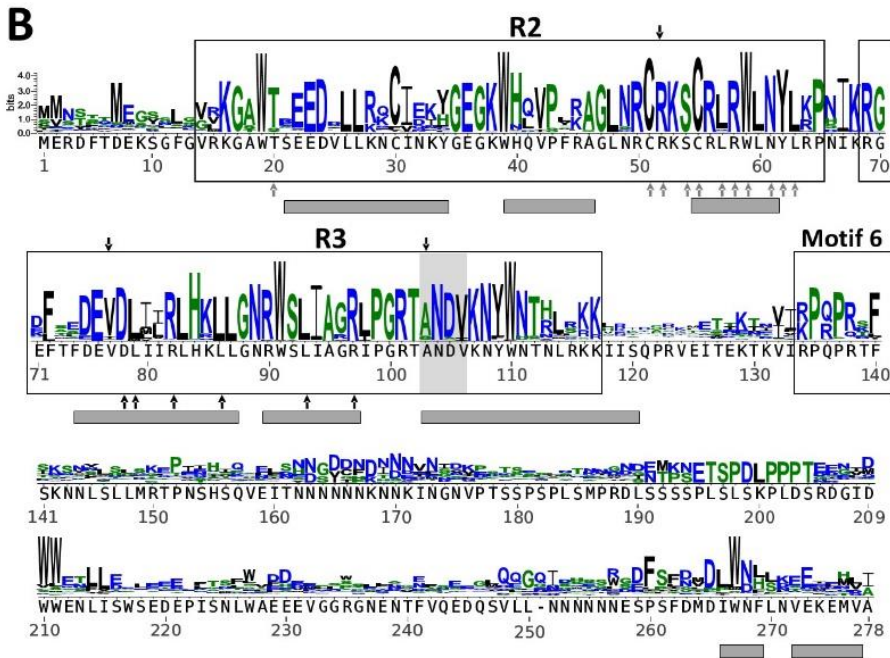

Figure 4. Similarity between Ribes MYB10 and other anthocyanin-inducing MYB proteins. (A) A phylogenetic timetree of selected anthocyanin-inducing MYB (MYB10-like) proteins. MYBF1 and MYBPA1 are non-MYB10-like, they activate flavanol- and proanthocyanidin-synthesizing enzymes, respectively. All proteins are named exactly the same way as described in the Figure 3 legend. (B) Sequence logo representing an alignment of 117 MYB10-like proteins from different species of the Pentapetalae taxonomic branch. Motif 6 (originally defined as KPRPR[S/T]F) is specific to subgroup 6 of the MYB proteins [27]. Gray background and black arrows on the top of the sequence denote amino acids specific to anthocyanin regulators [28]. Gray arrows point to amino acids important to DNA binding [29]. Black arrows under the sequence point to the motif ([DE]Lx2[RK]x3Lx6Lx3R) involved in interaction with bHLH proteins [30]. Other annotations are the same as described in the Figure 3 legend.

\subsection{Expression of Anthocyanin Pathway Genes in Fruits of Different Ribes Cultivars}

To evaluate the expression of anthocyanin pathway genes, we designed oligonucleotide primer pairs targeting regions of genes for which sequence information was available for all analyzed species. Gene expression analysis was performed by qPCR using the same gene-specific primer pair used to analyze different species of the Ribes genus. To test whether cloned genes were differentially expressed in fruits with different anthocyanin contents, three cultivars containing high, medium or low quantities of fruit anthocyanins were selected from R. nigrum (Figure 5A) and R. uva-crispa (Figure 5B) species. One cultivar with high fruit anthocyanin content was selected from R. rubrum and R. aureum 
(Figure 5C) to verify whether the designed oligonucleotide primers are suitable for the analysis of these Ribes species.

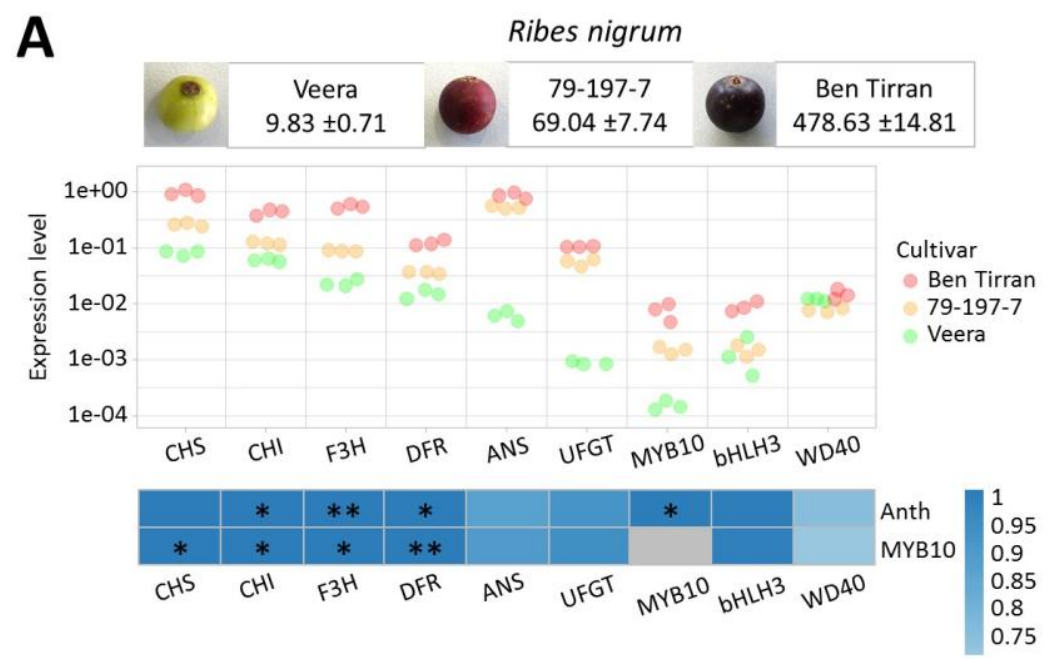

B

B Ribes uva-crispa
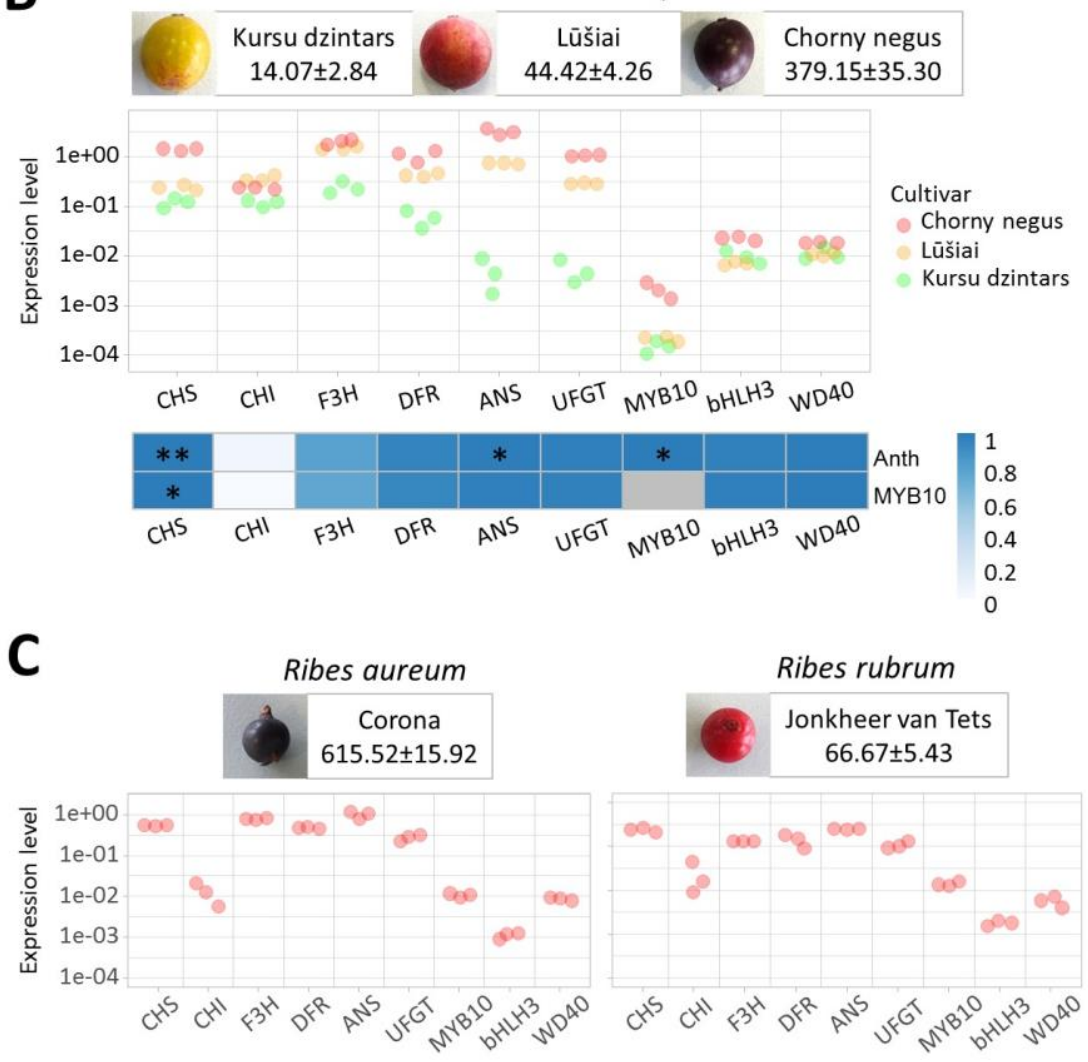

Figure 5. Expression of flavonoid pathway genes in fruits of different Ribes cultivars. For each species, ripe fruits with specified total anthocyanin content in $\mathrm{mg} / 100 \mathrm{~g}$ of fresh fruit weight are shown at the top, qPCR expression data in the middle and Pearson correlation analysis of expression levels of each gene and anthocyanin content (Anth) or MYB10 expression level at the bottom. Each point in the gene expression dot plots represents the average of three technical replicates. All expression data are presented on a logarithmic scale and normalized by the level of control gene expression. The statistical significance of Pearson correlation is denoted as: ${ }^{*}, p$ value $\leq 0.05 ;{ }^{* *}, p$ value $\leq 0.01$. (A) Three Ribes nigrum cultivars with different anthocyanin levels. (B) Three Ribes uva-crispa cultivars with different anthocyanin levels. (C) Ribes rubrum cultivar "Jonkheer van Tets" and Ribes aureum cultivar "Corona". 
Gene expression assay by qPCR showed that all genes encoding anthocyanin pathway enzymes were expressed at higher levels in fruits of the same species with higher anthocyanin levels (Figure 5A,B). Expression levels of genes encoding enzymes participating in the final stages of the anthocyanin pathway differed the most markedly. For example, expression levels of the ANS and UFGT genes differed by more than two to almost three levels of magnitude between cultivars with the highest and lowest anthocyanin content.

The expression levels of putative regulatory genes were generally lower. The expression level of the WD40 gene was almost constant in different species and cultivars with different anthocyanin levels. The expression of the $b H L H 3$ gene was more variable between different species and different cultivars. However, there was no clear difference in the expression levels of $b H L H 3$ between cultivars with lower anthocyanin content in both $R$. nigrum and R. uva-crispa. The relative expression levels of the MYB10 gene were not high in cultivars of R. nigrum and especially in cultivars of R. uva-crispa. Nevertheless, the expression of the MYB10 gene is obviously higher in cultivars with higher anthocyanin levels, and this can be seen more clearly in the case of R. nigrum (Figure 5A).

Pearson correlation analysis revealed positive correlation between the expression of almost all genes and the anthocyanin content, as well as with the expression of MYB10. However, although the highest differences between cultivars with lowest ant highest anthocyanin content can be seen in the expression of the ANS and UFGT genes, the statistical significance of correlation between the expression of these genes and anthocyanin content (or expression of MYB10) is not as high as for some other genes. This can be explained by the observation that the expression patterns of these two genes in different cultivars do not follow the patterns observed for the anthocyanin content or MYB10 expression. The highest difference in expression of ANS and UFGT is between cultivars with lower anthocyanin content, whereas the highest difference in anthocyanin content (and expression of MYB10) is between cultivars with higher anthocyanin content.

\subsection{Transient Expression of Ribes rubrum MYB10 and bHLH3 Regulatory Genes in Leaves of Nicotiana Plants}

The presence of specific domains and motifs, overall sequence homology to their counterparts in other species (Figures 3 and 4) and the expression pattern (Figure 5A,B) of cloned MYB10 genes in cultivars with varying anthocyanin levels suggest that they are functional regulators of the anthocyanin biosynthesis pathway. However, the ability to induce or enhance anthocyanin synthesis in some experimental system could show substantial proof of their function. Gene overexpression in Nicotiana tabacum leaves is one such system. Tobacco leaves possess functional genes of the anthocyanin pathway and, probably, a sufficient level of WD40 protein, because no significant differences of its expression are observed between green leaves and anthocyanin-accumulating transgenic tobacco leaves [31], and the addition of a heterologous WD40 gene also did not observably influence anthocyanin accumulation induced by heterologous $M Y B$ and $b H L H$ genes in previous infiltration experiments [32]. Thus, the expression of relevant MYB and bHLH proteins from infiltrated heterologous genes can lead to the formation of an active MBW complex, which can upregulate native tobacco anthocyanin pathway genes. We analyzed the ability of RrbHLH3 and RrMYB10 genes to induce anthocyanin synthesis while transiently expressed in two Nicotiana species. RrMYB10 induced clear red patches after infiltration into $N$. tabacum leaves even without the simultaneous infiltration of any bHLH gene (Figure 6A, top left).

To test the ability of R. rubrum bHLH3 to assist MYB proteins in inducing anthocyanin synthesis, RrbHLH3 was infiltrated together with RrMYB10 or A. thaliana MYB10-like regulator PAP1. In these experiments, the effect of RrbHLH3 infiltration was hardly noticeable because RrMYB10 and PAP1 induce strong anthocyanin synthesis by themselves (Figure 6A, top right vs. top left). Thus, in a subsequent experiment, we infiltrated RrbHLH3 together with another MYB, P. avium anthocyanin regulator PaMYB10.1-3. PaMYB10.1-3 alone is unable to induce anthocyanin synthesis in N. tabacum and only moderate anthocyanin synthesis in N. benthamiana, but is able to induce strong anthocyanin synthesis in both Nicotiana plants when infiltrated together with the relevant $b H L H$ gene. The results showed that RrbHLH3 is able to function as a coactivator: in N. tabacum, such a pair of genes induced 
anthocyanin synthesis (Figure 6A, middle), whereas in N. benthamiana, anthocyanin synthesis was enhanced (Figure 6A, bottom). When infiltrated alone, RrbHLH3 was unable to induce anthocyanin synthesis, like other tested bHLH genes.
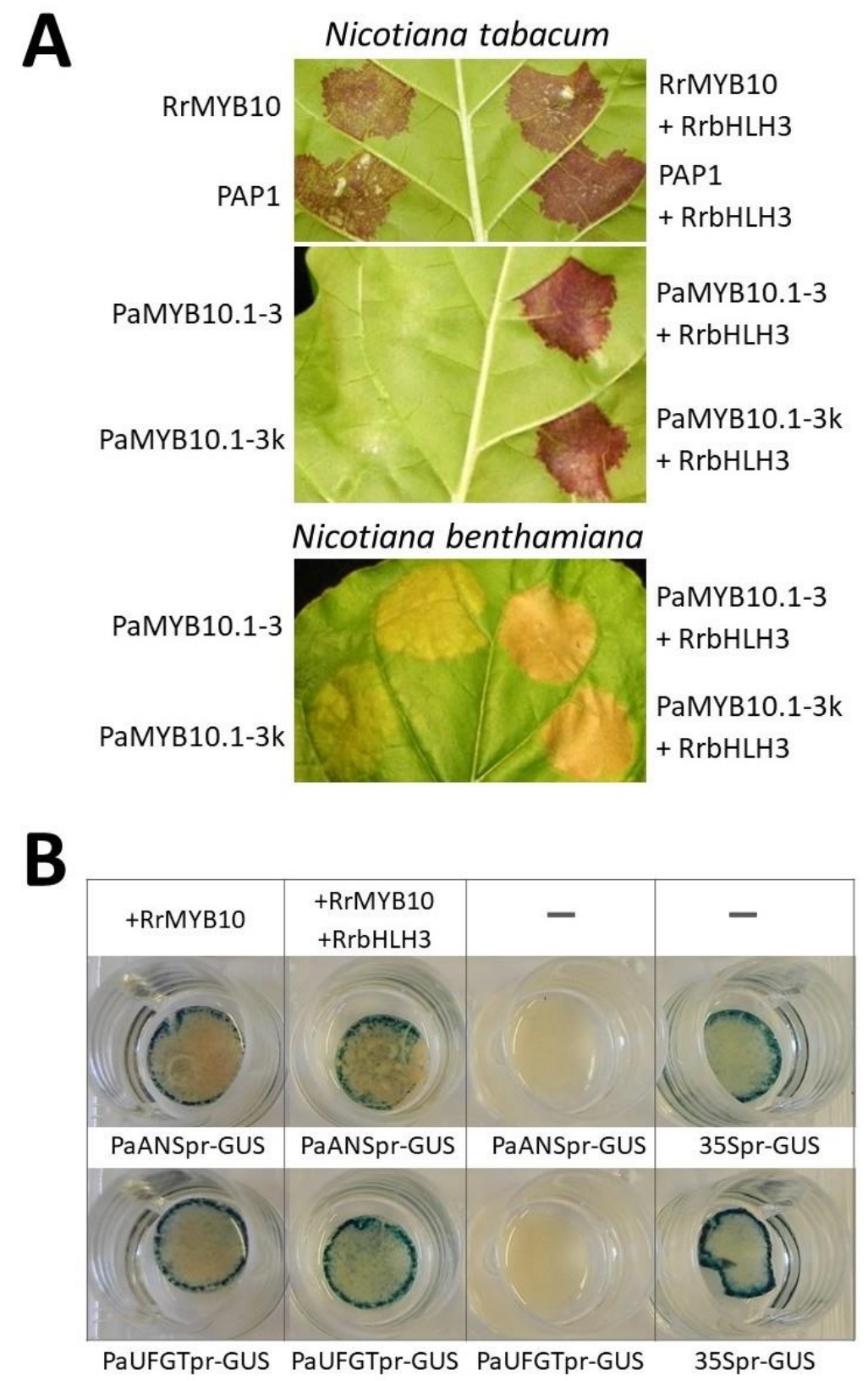

Figure 6. Transient expression of Ribes rubrum anthocyanin synthesis regulators in leaves of N. tabacum and N. benthamiana species. (A) The induction of anthocyanin synthesis by infiltrated regulatory genes. The comparison of the inducing capabilities of RrMYB10 and AtPAP1, and the effect of simultaneous infiltration of RrbHLH3 (top). The effect on the inducing capabilities of PaMYB10.1-3 elicited by the infiltration of RrbHLH3 in N. tabacum (middle) and N. benthamiana (bottom) leaves. PaMYB10.1-3k denotes an expression vector for cDNA of the PaMYB10.1-3 gene. (B) The reporter activation assay in $N$. tabacum leaves. GUS ( $\beta$-glucuronidase) expression cassette under the control of P. avium UFGT or ANS promoter was infiltrated together with RrMYB10 or both RrMYB10 and RrbHLH3. Single GUS expression cassette under the control of PaUFGT or PaANS promoter served as a negative control, whereas single GUS expression cassette under the control of cauliflower mosaic virus 35S promoter served as a positive control. 
To test the ability of RrMYB10 and RrbHLH3 genes to induce anthocyanin pathway components from the rosid clade, we cloned promoters of P. avium ANS and UFGT genes. The promoters were fused with the Escherichia coli $\beta$-glucuronidase coding sequence, and such expression vectors were infiltrated together with the analyzed genes into the same $N$. tabacum system. The results were the same as in the anthocyanin induction experiments: RrMYB10 alone was able to activate PaANS and PaUFGT promoters, whereas supplemented RrbHLH3 slightly enhanced this induction (Figure 6B).

\section{Discussion}

In this work, we cloned anthocyanin pathway genes and their regulators from several species of the Ribes genus. Since this genus, as well as the whole Saxifragales clade, has not been extensively analyzed at the molecular biology level, the sequence information itself presents scientific value. In general, anthocyanin pathway enzymes show a high degree of homology with the counterparts from other species, especially at the amino acid level (Figure 2 and Figures S1-S9). The conservancy of the WD40 protein was also expected. This only constant member of the variable regulatory MBW complex has a highly conserved structure because it participates in the regulation of various processes in various tissues by not defining functional specificity of different complexes [33-35].

The second member of the regulatory complex can be any protein belonging to subgroup IIIf [36] of the bHLH protein family $[37,38]$. In Arabidopsis, these are the TT8, EGL3/GL3 and MYC1 proteins. The first member of the bHLH subgroup IIIf proteins is Arabidopsis TT8 and its homologs, named bHLH3 in most Rosaceae species, and An1 in tobacco (the branch named bHLH3 in Figure 3A). MBW complexes containing bHLH3-like proteins activate anthocyanin synthesis in plant tissues [39,40]. The functions of other subgroup IIIf bHLH proteins are less clear. Closely related proteins EGL3 and GL3 upregulate anthocyanin synthesis in Arabidopsis, but their homologs in other species, named JAF13 in tobacco and varyingly in other species (the branch named bHLH43 in Figure 3A), have not been studied extensively. According to recent data, tobacco JAF13 activates anthocyanin synthesis indirectly, by activating the expression of NtAn1 [39]. The third bHLH protein, which can take part in the MBW complex, is Arabidopsis MYC1, named bHLH33 in most Rosaceae and some other species (the branch named bHLH33 in Figure 3A). This bHLH protein shows activating [41] or repressing [32] activities in different rosid species and is absent in the asterid clade. We cloned the bHLH3 protein as the most obvious activating bHLH member of the MBW complex.

The diversity of MYB proteins entering the MBW complex is large and embraces activators as well as repressors, proteins with different structures that are differentially expressed and active only in specific organs, tissues and developmental stages and those that regulate different branches of the flavonoid pathway [42,43]. Sequence analysis of cloned MYB genes (Figure 4) reveals that they encode R2R3 class subgroup 6 proteins [27]. Proteins belonging to this group there named MYB10 in Rosaceae $[28,41]$ and in some other species $[44,45]$. It is well established that MYB10-like proteins activate the anthocyanin pathway in fruits [12]. The grouping of MYB10-like sequences belonging to the asterid, rosid and Saxifragales clades is not so strict, probably because there are differently specialized anthocyanin-inducing MYB10-like proteins in a single organism, whereas the phylogenetic tree is composed of arbitrary selected subgroup 6 sequences from each organism (Figure 4A).

Most flavonoid pathway enzymes in many plants are encoded by more than one gene, and different genes for the same enzyme can be differently regulated temporarily as well as spatially. Data generated by transcriptomic studies on anthocyanin synthesis regulation in bayberry, black raspberry and sweet cherry reveal that the expression of at least one gene encoding both late and early flavonoid pathway enzymes strongly correlates with anthocyanin level [16-18]. Expression analysis of anthocyanin pathway genes in fruits of different Ribes genus cultivars revealed that the activities of most enzyme-coding genes strongly correlate with fruit anthocyanin content (Figure 5). Thus, cloned Ribes cDNAs represent the enzyme genes responsible for anthocyanin accumulation in fruits, although there may be additional genes active in different tissues or causing the accumulation of flavonols in earlier stages. 
In contrast to enzyme-coding genes, WD40 showed a similar expression level in all cultivars. This is in agreement with results obtained for P. avium, in which the expression of WD40 was almost constant in different cultivars and fruit maturation stages [32,46]. The expression of bHLH3 was more variable and to some degree correlated with anthocyanin content. In the fruits of other plants, bHLH3 expression is usually variable and in some plants positively correlates with anthocyanin production, such as in Lycium ruthenicum [47] and Brassica oleracea [48], but not in others, such as Prunus avium $[32,46]$. The expression of MYB10 strongly and reliably correlated with anthocyanin content in both R. nigrum and R. uva-crispa, but was not as high as in some other plants, where its level sometimes reached that of the highly expressed enzyme genes. The differences in the expression of MYB10 in the three cultivars of each species were not exactly replicated by the differences in expression of the late anthocyanin pathway genes ANS and UFGT. This may suggest that ANS and UFGT genes are also regulated by some other regulatory factors, not identified in this work.

Transient expression assays in tobacco leaves confirmed bHLH3 as a coactivator protein and revealed that RrMYB10 can induce anthocyanin synthesis without the addition of any helper bHLH. Although many anthocyanin-inducing MYB proteins require the addition of some external bHLH, some MYB10-like proteins, for example, MYB1 from lychee fruit [49] or MYB110 from kiwifruit petals [50], are also able to induce anthocyanin synthesis without any external bHLH proteins. In the latter case, an external MYB protein seems to be able to induce the expression of relevant tobacco bHLH proteins [39]. Thus, the induction of anthocyanin synthesis in N. tabacum without any helper bHLH proteins reveals the strong activating capabilities of RrMYB10 and suggests its ability to induce the expression of endogenous tobacco bHLH genes.

Sequence information obtained in this work can be applied in Ribes breeding programs and further anthocyanin research. With gene sequences of anthocyanin pathway enzymes and their main regulators available, it is possible to search for the exact sequence differences responsible for different anthocyanin levels and composition in various cultivars. This can accelerate the development of varieties with desired traits by applying molecular genetics methods to conventional breeding or by the use of genetic engineering. With verified qPCR primers available, which are suitable for the analysis of most important Ribes species, molecular methods can be used to analyze gene expression in response to various environmental stimuli and other factors. The analyzed anthocyanin regulators, especially RrMYB10, which has strong anthocyanin-inducing capabilities, can be exploited in manipulating anthocyanin synthesis in heterologous plant systems.

\section{Materials and Methods}

\subsection{Plant Material}

Fully mature fruits, which met the criteria of commercial ripeness defined individually for each cultivar (relevant color, size and taste), were collected from R. nigrum cultivars "Ben Tirran", "Veera" and clone "79-197-7"; R. uva-crispa cultivars "Chorny negus", "Lūšiai" and "Kursu dzintars"; R. rubrum cultivar "Jonkheer van Tets", and R. aureum cultivar "Corona". Samples representing each cultivar consisted of twenty berries collected from five different plants. Bushes were grown at the Institute of Horticulture of Lithuanian Research Centre for Agriculture and Forestry, Babtai under field conditions (lat: 55.0000, long: 23.8000; elevation: $120 \mathrm{~m}$ ). After harvesting, fruits were immediately frozen in liquid nitrogen and stored at $-80^{\circ} \mathrm{C}$ for subsequent analysis. N. tabacum and $N$. benthamiana were used for transient transformation experiments. Prunus avium cultivars "Irema BS" and "Kitayanka" were used for the isolation of promoter sequences. Measurement of the total anthocyanin concentration was performed as described earlier [32]. 


\subsection{DNA Isolation, $P C R$, Gene Cloning and Sequencing}

DNA for gene cloning was isolated from shoots of R. nigrum, R. uva-crispa, R. aureum and P. avium plants using a GeneJET Plant Genomic DNA Purification Mini Kit (Thermo Fisher Scientific, Lithuania, Vilnius). Gene fragments were isolated by PCR using partially degenerated primers designed to match the consensus sequence derived by aligning homologous sequences from the Pentapetalae taxonomic branch (Table S1). Sequences for the alignments were obtained by analyzing NCBI nucleic acid (NR), transcript shotgun assembly (TSA) and whole genome sequence (WGS) databases. PCR reactions were performed with Phusion High-Fidelity DNA Polymerase (Thermo Fisher Scientific, Lithuania, Vilnius). All PCR fragments were cloned and sequenced.

\subsection{Isolation of $R N A, c D N A$ Synthesis and $R A C E$}

Pieces of $3-5$ berries containing $0.5 \mathrm{~g}$ of mesocarp and exocarp tissue from each Ribes cultivar were finely ground in liquid nitrogen and isolated with a Sigma Spectrum Plant Total RNA Kit. The cDNA synthesis was performed with a RevertAid H Minus First Strand cDNA Synthesis Kit (Thermo Fisher Scientific, Lithuania, Vilnius). For qPCR analysis, RNA samples were treated with RNase-free DNase I before cDNA synthesis. The $3^{\prime}$ - and 5'-RACE were performed to obtain sequence information for regions outside those cloned with degenerated primers and to obtain full coding frames of genes used in functional experiments. For 3'-RACE, cDNA was synthesized using primer 3-RACE-CDS-A. For $5^{\prime}$-RACE and $\mathrm{qPCR}, \mathrm{CDNA}$ was synthesized using polyT primer. A template-switching reaction for $5^{\prime}$-RACE was performed with SMART-C3 oligo using Thermo Fisher Scientific enzymes and buffers, similarly as described previously [51]. Direct and nested RACE PCR was performed using a gene-specific primer together with primer mix UPM and primer NUP-A, respectively. All primer sequences for RACE have been published previously [32]. All obtained sequences were submitted to the European Nucleotide Archive (ENA) under submission numbers LN736309-LN736346.

\subsection{Gene Expression Analysis}

All qPCR assays were performed according to MIQE Guidelines [52]. A Rotor-Gene Q system (Qiagen) was used to perform quantitative real time PCR (qPCR) amplification. All reactions were performed with Maxima SYBR Green/ROX qPCR Master Mix (Thermo Fisher Scientific, Lithuania, Vilnius) in a $20 \mu \mathrm{L}$ volume with the following cycling parameters: $10 \mathrm{~min}$ at $95^{\circ} \mathrm{C}, 40$ cycles for $15 \mathrm{~s}$ at $95^{\circ} \mathrm{C}$ and $60 \mathrm{~s}$ at $60^{\circ} \mathrm{C}$; melting curve detection from $60^{\circ} \mathrm{C}$ to $95^{\circ} \mathrm{C}$. Three biological samples, each composed of five berries, were analyzed, and three technical replications were performed. Data were analyzed with Rotor-Gene $Q$ Series software version 2.1.0 using the $\Delta \Delta C \mathrm{Ct}$ method [53]. The expression level was normalized to Ribes actin (ENA LN736321). The qPCR reaction products were verified by melting curve analysis, electrophoresis and sequencing. All qPCR primer sequences are listed in Table S2.

\subsection{Construction of Plasmids for Transient Gene Expression Assays}

The RrMYB10 and RrbHLH3 genes, as well as $\sim 1.5 \mathrm{~kb}$ fragments of both Prunus avium promoters (PaANS and PaUFGT), were PCR amplified with Phusion polymerase and cloned into a pJET1.2 vector. NruI/NotI-digested RrMYB10 and RrbHLH were recloned into SmiI/NotI-digested vector pENTR/D (Thermo Fisher Scientific, Lithuania, Vilnius). Finally, the RrMYB10 and RrbHLH genes were inserted into a destination vector pAUGLR by recombination using Gateway LR clonase II enzyme mix (Thermo Fisher Scientific, Lithuania, Vilnius). EcoRV/SalI-digested PaANS and EcoRI/XhoI-digested PaUFGT promoter fragments were cloned into Ecl136I//XhoI-digested vector pAUGLR. A gene encoding E. coli $\beta$-glucuronidase was inserted downstream of the cloned promoters by recombination using Gateway LR clonase II enzyme mix. Primers used for gene cloning and the construction of expression vectors are provided in Table S3. Plasmids for the expression of AtPAP1, PaMYB10.1-3 and PaMYB10.1-3k 
were constructed as described previously [32]. For infiltration experiments, all final plasmids were electroporated into Agrobacterium tumefaciens GV3101 strain.

\subsection{Transient Gene Expression Assays and Plant Transformation}

$N$. tabacum and $N$. benthamiana plants were grown in soil at $25{ }^{\circ} \mathrm{C}$ in a room with controlled environment under long day conditions of $16 \mathrm{~h}$ of white light and $8 \mathrm{~h}$ of dark. An A. tumefaciens suspension was syringe infiltrated into the abaxial side of the leaves of five- to six-week-old plants. Overnight cultures of $A$. tumefaciens were grown in LB medium with rifampicin and spectinomycin, diluted 500-fold with LB containing $10 \mathrm{mM}$ MES (2-(N-morpholino)ethanesulfonic acid), and grown overnight till $\mathrm{A} 600=1.2-1.6$. After sedimentation by centrifugation for $20 \mathrm{~min}$ at $3000 \times g$, the cells were suspended in infiltration buffer (10 mM MES pH 5.7, $10 \mathrm{mM} \mathrm{MgCl} 2,500 \mu \mathrm{M}$ acetosyringone) till $\mathrm{A} 600=1.8$ and incubated overnight at room temperature.

A helper strain containing a silencing suppressor from plum pox virus (PPV HC-Pro) [54] was used in all infiltration experiments. When testing several expression cassettes in a single infiltration, transformed Agrobacterium strains were mixed at equal ratios. Coloration around infiltration zones, indicating anthocyanin synthesis, was observable on the abaxial side of $N$. tabacum leaves and the adaxial side of $N$. benthamiana leaves starting from three days post infiltration. Photographs were always taken seven days post infiltration. In all experiments, the expression of infiltrated genes was verified by RT-PCR.

\subsection{Promoter Activity Assays Using Reporter Gene Expression}

Promoter activity assays were performed by infiltrating promoter::GUS plasmids into N tabacum leaves, as already described. Leaf discs with the infiltration point at the center were cut out using a $12 \mathrm{~mm}$ diameter cork borer thirteen days post infiltration. Isolated leaf discs were placed abaxial side up into a 24-well plate containing X-gluc buffer solution $(0.25 \mathrm{mg} / \mathrm{mL}$ X-gluc (5-Bromo-4-chloro-1H-indol-3-yl $\beta$-D-glucopyranosiduronic acid) in DMSO, $0.5 \mathrm{mM}$ potassium ferrocyanide $(\mathrm{K} 4 \mathrm{Fe}(\mathrm{CN}) 63 \mathrm{H} 2 \mathrm{O}), 0.5 \mathrm{mM}$ potassium ferricyanide ( $\mathrm{K} 3[\mathrm{Fe}(\mathrm{CN}) 6]), 0.01 \%$ Tween $20,100 \mathrm{mM}$ sodium phosphate buffer), and vacuum applied for $12 \mathrm{~min}$. Samples were incubated in the dark at $37^{\circ} \mathrm{C}$ for $24-48 \mathrm{~h}$ until the distinct blue staining appeared. Finally, leaf discs were washed several times in a series of $70 \%$ and $80 \%(v / v)$ aqueous ethanol solutions until the chlorophyll was removed.

\subsection{Bioinformatics Analysis}

Sequences of plant anthocyanin pathway genes were obtained by BLAST searching NCBI NR, WGS and TSA databases (http://www.ncbi.nlm.nih.gov). Multiple sequence alignments were accomplished with Clustal Omega [55], and phylogenetic trees were created with MEGA version 7 [56] using the neighbor-joining method with 1500 bootstrap repetitions. Sequence logos were created with WebLogo 3 [57]. Protein domains and amino acids with known functionality were annotated by NCBI's conserved domain database (CDD) [58]; secondary structure was predicted using PSIPRED [59].

Supplementary Materials: The following are available online at http://www.mdpi.com/2223-7747/9/9/1078/s1, Figure S1: Phylogenetic tree of selected UFGT proteins, Figure S2: Phylogenetic tree of selected ANS proteins, Figure S3: Phylogenetic tree of selected DFR proteins, Figure S4: Phylogenetic tree of selected F3H proteins, Figure S5: Phylogenetic tree of selected CHI proteins, Figure S6: Phylogenetic tree of selected CHS proteins, Figure S7: Phylogenetic tree of selected WD40 proteins, Figure S8: Phylogenetic tree of selected bHLH3 proteins, Figure S9: Phylogenetic tree of selected MYB10 proteins, Table S1: Partially degenerated primers for used to clone fragments of Ribes anthocyanin pathway genes, Table S2: qPCR primers used in the study, Table S3: Primers for construction of plasmids for transient gene expression and promoter cloning from the genome.

Author Contributions: Conceptualization, R.R. (Raimundas Ražanskas); Data curation, R.R. (Raimundas Ražanskas); Funding acquisition, V.K.; Investigation, P.S., A.R., U.S., V.K., E.D., V.B. and R.R. (Raimundas Ražanskas); Methodology, A.R. and R.R. (Raimundas Ražanskas); Project administration, V.K.; Resources, V.S., T.Š. and R.R. (Rytis Rugienius); Supervision, A.R., V.K. and R.R. (Raimundas Ražanskas); Visualization, R.R. (Raimundas Ražanskas); Writing-original draft, R.R. (Raimundas Ražanskas); Writing-review and editing, R.R. (Raimundas Ražanskas). All authors have read and agreed to the published version of the manuscript. 
Funding: This research was funded by the Research Council of Lithuania, grant number SVE-06/2012.

Conflicts of Interest: The authors declare no conflict of interest.

\section{References}

1. Hummer, K.E.; Barney, D.L. Currants. Horttechnology 2002, 12, 377-387. [CrossRef]

2. Cortez, R.E.; Gonzalez de Mejia, E. Blackcurrants (Ribes nigrum): A Review on Chemistry, Processing, and Health Benefits. J. Food Sci. 2019, 84, 2387-2401. [CrossRef] [PubMed]

3. Gopalan, A.; Reuben, S.C.; Ahmed, S.; Darvesh, A.S.; Hohmann, J.; Bishayee, A. The health benefits of blackcurrants. Food Funct. 2012, 3, 795-809. [CrossRef] [PubMed]

4. Anisimovienè, N.; Jankauskienè, J.; Jodinskienė, M.; Bendokas, V.; Stanys, V.; Šikšnianas, T. Phenolics, antioxidative activity and characterization of anthocyanins in berries of blackcurrant interspecific hybrids. Acta Biochim. Pol. 2013, 60, 767-772. [CrossRef]

5. Vagiri, M.; Ekholm, A.; Öberg, E.; Johansson, E.; Andersson, S.C.; Rumpunen, K. Phenols and ascorbic acid in black currants (Ribes nigrum L.): Variation due to genotype, location, and year. J. Agric. Food Chem. 2013, 61, 9298-9306. [CrossRef]

6. Bendokas, V.; Skemiene, K.; Trumbeckaite, S.; Stanys, V.; Passamonti, S.; Borutaite, V.; Liobikas, J. Anthocyanins: From plant pigments to health benefits at mitochondrial level. Crit. Rev. Food Sci. Nutr. 2019, 1-14. [CrossRef]

7. Slimestad, R.; Solheim, H. Anthocyanins from black currants (Ribes nigrum L.). J. Agric. Food Chem. 2002, 50, 3228-3231. [CrossRef]

8. Tian, Y.; Laaksonen, O.; Haikonen, H.; Vanag, A.; Ejaz, H.; Linderborg, K.; Karhu, S.; Yang, B. Compositional Diversity among Blackcurrant (Ribes nigrum) Cultivars Originating from European Countries. J. Agric. Food Chem. 2019, 67, 5621-5633. [CrossRef]

9. Rubinskiene, M.; Jasutiene, I.; Venskutonis, P.R.; Viskelis, P. HPLC determination of the composition and stability of blackcurrant anthocyanins. J. Chromatogr. Sci. 2005, 43, 478-482. [CrossRef]

10. Deluc, L.; Barrieu, F.; Marchive, C.; Lauvergeat, V.; Decendit, A.; Richard, T.; Carde, J.P.; Merillon, J.M.; Hamdi, S. Characterization of a grapevine R2R3-MYB transcription factor that regulates the phenylpropanoid pathway. Plant Physiol. 2006, 140, 499-511. [CrossRef]

11. Saito, K.; Yonekura-Sakakibara, K.; Nakabayashi, R.; Higashi, Y.; Yamazaki, M.; Tohge, T.; Fernie, A.R. The flavonoid biosynthetic pathway in Arabidopsis: Structural and genetic diversity. Plant Physiol. Biochem. 2013, 72, 21-34. [CrossRef] [PubMed]

12. Takos, A.M.; Jaffé, F.W.; Jacob, S.R.; Bogs, J.; Robinson, S.P.; Walker, A.R. Light-induced expression of a MYB gene regulates anthocyanin biosynthesis in red apples. Plant Physiol. 2006, 142, 1216-1232. [CrossRef] [PubMed]

13. Saigo, T.; Wang, T.; Watanabe, M.; Tohge, T. Diversity of anthocyanin and proanthocyanin biosynthesis in land plants. Curr. Opin. Plant Biol. 2020, 55, 93-99. [CrossRef] [PubMed]

14. Xu, W.; Dubos, C.; Lepiniec, L. Transcriptional control of flavonoid biosynthesis by MYB-bHLH-WDR complexes. Trends Plant Sci. 2015, 20, 176-185. [CrossRef]

15. Lloyd, A.; Brockman, A.; Aguirre, L.; Campbell, A.; Bean, A.; Cantero, A.; Gonzalez, A. Advances in the MYB-bHLH-WD Repeat (MBW) Pigment Regulatory Model: Addition of a WRKY Factor and Co-option of an Anthocyanin MYB for Betalain Regulation. Plant Cell Physiol. 2017, 58, 1431-1441. [CrossRef]

16. Wei, H.; Chen, X.; Zong, X.; Shu, H.; Gao, D.; Liu, Q. Comparative transcriptome analysis of genes involved in anthocyanin biosynthesis in the red and yellow fruits of sweet cherry (Prunus avium L.). PLoS ONE 2015, 10, e0121164. [CrossRef]

17. Feng, C.; Chen, M.; Xu, C.; Bai, L.; Yin, X.; Li, X.; Allan, A.C.; Ferguson, I.B.; Chen, K. Transcriptomic analysis of Chinese bayberry (Myrica rubra) fruit development and ripening using RNA-Seq. BMC Genom. 2012, 13, 19. [CrossRef]

18. Hyun, T.K.; Lee, S.; Rim, Y.; Kumar, R.; Han, X.; Lee, S.Y.; Lee, C.H.; Kim, J.-Y. De-novo RNA sequencing and metabolite profiling to identify genes involved in anthocyanin biosynthesis in Korean black raspberry (Rubus coreanus Miquel). PLoS ONE 2014, 9, e88292. [CrossRef]

19. Zhao, D.; Tang, W.; Hao, Z.; Tao, J. Identification of flavonoids and expression of flavonoid biosynthetic genes in two coloured tree peony flowers. Biochem. Biophys. Res. Commun. 2015, 459, 450-456. [CrossRef] 
20. Jia, N.; Shu, Q.Y.; Wang, L.S.; Du, H.; Xu, Y.J.; Liu, Z.A. Analysis of petal anthocyanins to investigate coloration mechanism in herbaceous peony cultivars. Sci. Hortic. 2008, 117, 167-173. [CrossRef]

21. Qi, Y.; Zhou, L.; Han, L.; Zou, H.; Miao, K.; Wang, Y. PsbHLH1, a novel transcription factor involved in regulating anthocyanin biosynthesis in tree peony (Paeonia suffruticosa). Plant Physiol. Biochem. 2020, 154, 396-408. [CrossRef] [PubMed]

22. Zhang, X.; Xu, Z.; Yu, X.; Zhao, L.; Zhao, M.; Han, X.; Qi, S. Identification of Two Novel R2R3-MYB Transcription factors, PsMYB114L and PsMYB12L, Related to Anthocyanin Biosynthesis in Paeonia suffruticosa. Int. J. Mol. Sci. 2019, 20, 1055. [CrossRef] [PubMed]

23. Gu, Z.; Zhu, J.; Hao, Q.; Yuan, Y.-W.; Duan, Y.-W.; Men, S.; Wang, Q.; Hou, Q.; Liu, Z.-A.; Shu, Q.; et al. A Novel R2R3-MYB Transcription Factor Contributes to Petal Blotch Formation by Regulating Organ-Specific Expression of PsCHS in Tree Peony (Paeonia suffruticosa). Plant Cell Physiol. 2019, 60, 599-611. [CrossRef] [PubMed]

24. Ben-Simhon, Z.; Judeinstein, S.; Trainin, T.; Harel-Beja, R.; Bar-Ya'akov, I.; Borochov-Neori, H.; Holland, D. A "White" Anthocyanin-less Pomegranate (Punica granatum L.) Caused by an Insertion in the Coding Region of the Leucoanthocyanidin Dioxygenase (LDOX; ANS) Gene. PLoS ONE 2015, 10, e0142777. [CrossRef] [PubMed]

25. Appelhagen, I.; Jahns, O.; Bartelniewoehner, L.; Sagasser, M.; Weisshaar, B.; Stracke, R. Leucoanthocyanidin Dioxygenase in Arabidopsis thaliana: Characterization of mutant alleles and regulation by MYB-BHLH-TTG1 transcription factor complexes. Gene 2011, 484, 61-68. [CrossRef] [PubMed]

26. Rafique, M.Z.; Carvalho, E.; Stracke, R.; Palmieri, L.; Herrera, L.; Feller, A.; Malnoy, M.; Martens, S. Nonsense Mutation Inside Anthocyanidin Synthase Gene Controls Pigmentation in Yellow Raspberry (Rubus idaeus L.). Front. Plant Sci. 2016, 7, 1892. [CrossRef] [PubMed]

27. Stracke, R.; Werber, M.; Weisshaar, B. The R2R3-MYB gene family in Arabidopsis thaliana. Curr. Opin. Plant Biol. 2001, 4, 447-456. [CrossRef]

28. $\quad$ Lin-Wang, K.; Bolitho, K.; Grafton, K.; Kortstee, A.; Karunairetnam, S.; McGhie, T.K.; Espley, R.V.; Hellens, R.P.; Allan, A.C. An R2R3 MYB transcription factor associated with regulation of the anthocyanin biosynthetic pathway in Rosaceae. BMC Plant Biol. 2010, 10, 50. [CrossRef]

29. Feller, A.; Machemer, K.; Braun, E.L.; Grotewold, E. Evolutionary and comparative analysis of MYB and bHLH plant transcription factors. Plant J. 2011, 66, 94-116. [CrossRef]

30. Zimmermann, I.M.; Heim, M.A.; Weisshaar, B.; Uhrig, J.F. Comprehensive identification of Arabidopsis thaliana MYB transcription factors interacting with R/B-like BHLH proteins. Plant J. 2004, 40, 22-34. [CrossRef]

31. Zong, Y.; Li, S.; Xi, X.; Cao, D.; Wang, Z.; Wang, R.; Liu, B. Comprehensive Influences of Overexpression of a MYB Transcriptor Regulating Anthocyanin Biosynthesis on Transcriptome and Metabolome of Tobacco Leaves. Int. J. Mol. Sci. 2019, 20, 5123. [CrossRef] [PubMed]

32. Starkevic, P.; Paukstyte, J.; Kazanaviciute, V.; Denkovskiene, E.; Stanys, V.; Bendokas, V.; Siksnianas, T.; Razanskiene, A.; Razanskas, R. Expression and Anthocyanin Biosynthesis-Modulating Potential of Sweet Cherry (Prunus avium L.) MYB10 and bHLH Genes. PLoS ONE 2015, 10, e0126991. [CrossRef] [PubMed]

33. Tian, H.; Wang, S. TRANSPARENT TESTA GLABRA1, a Key Regulator in Plants with Multiple Roles and Multiple Function Mechanisms. Int. J. Mol. Sci. 2020, 21, 4881. [CrossRef] [PubMed]

34. Zhang, B.; Schrader, A. TRANSPARENT TESTA GLABRA 1-Dependent Regulation of Flavonoid Biosynthesis. Plants 2017, 6, 65. [CrossRef]

35. Xu, C.; Min, J. Structure and function of WD40 domain proteins. Protein Cell 2011, 2, 202-214. [CrossRef] [PubMed]

36. Heim, M.A.; Jakoby, M.; Werber, M.; Martin, C.; Weisshaar, B.; Bailey, P.C. The basic helix-loop-helix transcription factor family in plants: A genome-wide study of protein structure and functional diversity. Mol. Biol. Evol. 2003, 20, 735-747. [CrossRef]

37. Chezem, W.R.; Clay, N.K. Regulation of plant secondary metabolism and associated specialized cell development by MYBs and bHLHs. Phytochemistry 2016, 131, 26-43. [CrossRef]

38. Pires, N.; Dolan, L. Origin and diversification of basic-helix-loop-helix proteins in plants. Mol. Biol. Evol. 2010, 27, 862-874. [CrossRef] 
39. Montefiori, M.; Brendolise, C.; Dare, A.P.; Lin-Wang, K.; Davies, K.M.; Hellens, R.P.; Allan, A.C. In the Solanaceae, a hierarchy of bHLHs confer distinct target specificity to the anthocyanin regulatory complex. J. Exp. Bot. 2015, 66, 1427-1436. [CrossRef]

40. Xie, X.-B.; Li, S.; Zhang, R.-F.; Zhao, J.; Chen, Y.-C.; Zhao, Q.; Yao, Y.-X.; You, C.-X.; Zhang, X.-S.; Hao, Y.-J. The bHLH transcription factor MdbHLH3 promotes anthocyanin accumulation and fruit colouration in response to low temperature in apples. Plant Cell Environ. 2012, 35, 1884-1897. [CrossRef]

41. Espley, R.V.; Hellens, R.P.; Putterill, J.; Stevenson, D.E.; Kutty-Amma, S.; Allan, A.C. Red colouration in apple fruit is due to the activity of the MYB transcription factor, MdMYB10. Plant J. 2007, 49, 414-427. [CrossRef] [PubMed]

42. Allan, A.C.; Espley, R.V. MYBs Drive Novel Consumer Traits in Fruits and Vegetables. Trends Plant Sci. 2018, 23, 693-705. [CrossRef] [PubMed]

43. Dubos, C.; Stracke, R.; Grotewold, E.; Weisshaar, B.; Martin, C.; Lepiniec, L. MYB transcription factors in Arabidopsis. Trends Plant Sci. 2010, 15, 573-581. [CrossRef] [PubMed]

44. Elomaa, P.; Uimari, A.; Mehto, M.; Albert, V.A.; Laitinen, R.A.E.; Teeri, T.H. Activation of anthocyanin biosynthesis in Gerbera hybrida (Asteraceae) suggests conserved protein-protein and protein-promoter interactions between the anciently diverged monocots and eudicots. Plant Physiol. 2003, 133, 1831-1842. [CrossRef] [PubMed]

45. Palapol, Y.; Ketsa, S.; Lin-Wang, K.; Ferguson, I.B.; Allan, A.C. A MYB transcription factor regulates anthocyanin biosynthesis in mangosteen (Garcinia mangostana L.) fruit during ripening. Planta 2009, 229, 1323-1334. [CrossRef] [PubMed]

46. Alkio, M.; Jonas, U.; Declercq, M.; Van Nocker, S.; Knoche, M. Transcriptional dynamics of the developing sweet cherry (Prunus avium L.) fruit: Sequencing, annotation and expression profiling of exocarp-associated genes. Hortic. Res. 2014, 1, 11. [CrossRef] [PubMed]

47. Zeng, S.; Wu, M.; Zou, C.; Liu, X.; Shen, X.; Hayward, A.; Liu, C.; Wang, Y. Comparative analysis of anthocyanin biosynthesis during fruit development in two Lycium species. Physiol. Plant. 2014, 150, 505-516. [CrossRef]

48. Rahim, M.A.; Robin, A.H.K.; Natarajan, S.; Jung, H.-J.; Lee, J.; Kim, H.; Kim, H.-T.; Park, J.-I.; Nou, I.-S. Identification and Characterization of Anthocyanin Biosynthesis-Related Genes in Kohlrabi. Appl. Biochem. Biotechnol. 2018, 184, 1120-1141. [CrossRef]

49. Lai, B.; Li, X.J.; Hu, B.; Qin, Y.H.; Huang, X.M.; Wang, H.C.; Hu, G.B. LcMYB1 is a key determinant of differential anthocyanin accumulation among genotypes, tissues, developmental phases and ABA and light stimuli in Litchi chinensis. PLoS ONE 2014, 9, e86293. [CrossRef]

50. Fraser, L.G.; Seal, A.G.; Montefiori, M.; McGhie, T.K.; Tsang, G.K.; Datson, P.M.; Hilario, E.; Marsh, H.E.; Dunn, J.K.; Hellens, R.P.; et al. An R2R3 MYB transcription factor determines red petal colour in an Actinidia (kiwifruit) hybrid population. BMC Genom. 2013, 14, 28. [CrossRef]

51. Pinto, F.L.; Lindblad, P. A guide for in-house design of template-switch-based $5^{\prime}$ rapid amplification of cDNA ends systems. Anal. Biochem. 2010, 397, 227-232. [CrossRef] [PubMed]

52. Bustin, S.A.; Benes, V.; Garson, J.A.; Hellemans, J.; Huggett, J.; Kubista, M.; Mueller, R.; Nolan, T.; Pfaffl, M.W.; Shipley, G.L.; et al. The MIQE guidelines: Minimum information for publication of quantitative real-time PCR experiments. Clin. Chem. 2009, 55, 611-622. [CrossRef] [PubMed]

53. Rao, X.; Huang, X.; Zhou, Z.; Lin, X. An improvement of the $2^{\wedge}(-$ delta delta CT) method for quantitative real-time polymerase chain reaction data analysis. Biostat. Bioinform. Biomath. 2013, 3, 71-85.

54. Tenllado, F.; Barajas, D.; Vargas, M.; Atencio, F.A.; González-Jara, P.; Díaz-Ruíz, J.R. Transient expression of homologous hairpin RNA causes interference with plant virus infection and is overcome by a virus encoded suppressor of gene silencing. Mol. Plant Microbe Interact. 2003, 16, 149-158. [CrossRef]

55. Sievers, F.; Wilm, A.; Dineen, D.; Gibson, T.J.; Karplus, K.; Li, W.; Lopez, R.; McWilliam, H.; Remmert, M.; Söding, J.; et al. Fast, scalable generation of high-quality protein multiple sequence alignments using Clustal Omega. Mol. Syst. Biol. 2011, 7, 539. [CrossRef]

56. Kumar, S.; Stecher, G.; Tamura, K. MEGA7: Molecular Evolutionary Genetics Analysis Version 7.0 for Bigger Datasets. Mol. Biol. Evol. 2016, 33, 1870-1874. [CrossRef]

57. Crooks, G.E.; Hon, G.; Chandonia, J.-M.; Brenner, S.E. WebLogo: A sequence logo generator. Genome Res. 2004, 14, 1188-1190. [CrossRef] 
58. Yang, M.; Derbyshire, M.K.; Yamashita, R.A.; Marchler-Bauer, A. NCBI's Conserved Domain Database and Tools for Protein Domain Analysis. Curr. Protoc. Bioinform. 2020, 69, e90. [CrossRef]

59. Buchan, D.W.A.; Jones, D.T. The PSIPRED Protein Analysis Workbench: 20 years on. Nucleic Acids Res. 2019, 47, W402-W407. [CrossRef] 